\title{
A Comparison of the Tensile Strengths of Yucca Fiber Extracted by Microbial and Chemical Methods
}

\author{
Toye Ekunsanmi ${ }^{1}$ and Swapnil Tripathi ${ }^{2}$
}

1. Department of Mathematics and Natural Sciences, Biological Sciences, University of Wisconsin, Milwaukee - Washington County, USA

2. Department of Mathematics and Natural Sciences, Physics and Astronomy, University of Wisconsin, Milwaukee, Washington County, USA

\begin{abstract}
Leaves from greenhouse-grown Yucca plants were harvested and retted by microbial and chemical methods respectively. The dimensions and tensile strengths of the two sets of products were compared. While the fibers obtained by microbial retting had superior tensile strength, the chemically retted fibers were devoid of debris, whiter and generally more attractive. The method of retting should match the use to which the plant fibers are intended.
\end{abstract}

Key words: Bast fiber, wood fiber, nodes, tensile strength, retting.

\section{Introduction}

Many useful everyday materials are derived from plant fibers. Uses of fibers, include but are not limited to clothing, fire hoses, fish nets and cordage. Others include bowstrings, ropes, yarn, composite materials and burlap. Advantages of bio-fibers over synthetic fibers include renewability, being environmentally friendly, light weight and lack of dermal issues [1,2].

Fibers are mainly cellulose, which are more difficult to biodegrade than softer parts of the plant, but are nonetheless decomposed by a considerable number of microorganism which produce cellulase, the enzyme which digests cellulose. Such organisms, mainly fungi, are abundant in the soil, although in natural environments, the softer plant tissues are usually degraded first by microorganisms, leaving the fibers, which are more slowly degraded. Many plants have been used for fiber extraction, including jute, flax, hemp and ramie [3] In fact many of these plants are grown almost exclusively for their fibers. Obviously, there are many plants which qualify as

Corresponding author: Swapnil Tripathi, Ph.D., associate professor of physics and astronomy, research fields: theory of relativity, gravitational waves, interdisciplinary research. tripathi@uwm.edu. sources of fiber. However, the plants used for fiber extraction will depend on availability, cost of production and climatic factors for growth. Generally, when food plants such as corn, pineapple, sugarcane are used, the fibers are extracted from the by-products of their processing after other useful products have been extracted. Some other plants, easily classified as "weeds" have been used on experimental bases. Others in this category include Sansevaria, Agave and Pandanus and the common milkweed [3].

The method of fiber extraction may depend on the source but still follows the same general principle of freeing the fibers from other, usually softer plant tissues. The process by which the tissues are removed vary with the plant material, and the purpose to which the fibers are intended. The common name given to the process is retting. Retting is commonly done by employing microorganisms, this is called microbial retting. It could also be done mechanically by grinding the tissue, or by chemical means. More often than not, retting is accomplished by a combination of methods. For example, a combination of grinding and soaking in natural fresh water bodies is used to obtain fibers from many vine like plants. In microbial retting, the plant material is soaked in water, or buried in the soil, 
and the microbes are allowed to do carry out the process over time.

The plant, Yucca aloifolia, is a common ornamental plant in warmer climates. It is also planted indoors as such in colder climates, and will grow well given enough light, in addition to other growth conditions. The use of the plant for experimental fiber extraction seems to be quite popular. This may be due to the fact that it is easily propagated under controlled conditions in the greenhouse, thereby enabling specificity in the harvesting and processing methods. In this work, we extracted fibers from Yucca leaves by two microbial and chemical retting respectively. The goal is to determine the extent to which the fiber strength is affected by the method of retting.

\section{Materials and Methods}

\subsection{Microbial Retting}

Leaves were harvested from a potted Yucca plant in the greenhouse of the University of Wisconsin, Washington County. Leaves chosen for microbial and chemical retting were selected from whorls of the same age to ascertain uniformity in fiber content. Each leave was carefully cut as close as possible to the base using a sharp razor blade.

\subsection{Chemical Retting}

The Yucca leaves were laid on a hard surface and gently scraped with a blunt knife from base to apex to remove the cuticle and epidermal layers. The leaves were then boiled for 45 minutes in $11 \% \mathrm{NaOH}$, and carefully transferred to a container of $3 \%$ hydrogen peroxide in a tray and allowed to stand for 10 minutes. It was then decolorized in full strength household bleach for 5 minutes. Excess chemicals were removed by rinsing in several changes of tap water. The fibers were then allowed to dry in a fume chamber.

\subsection{Microbial Retting}

Leaves were harvested from a potted Yucca plant in the greenhouse of the University of Wisconsin,
Washington County. Leaves chosen for microbial and chemical retting were selected from whorls of close whorls, to ascertain uniformity in leaf age. Each leave was carefully cut as close as possible to the base using a sharp razor blade. The leaves for microbial retting were fully immersed without grinding or crushing in a plastic trough filled to $75 \%$ of its volume with deionized water and inoculated with a trowel-full of loamy garden soil containing some amount of decomposing leaves. This was intended to introduce possible microbes for retting. The leaves were left for 90 days with intermittent addition of de-ionized water as needed to prevent drying out.

At the end of the period, the partially decomposed leaves were carefully removed with a pair of forceps without bending or twisting. The decomposed parts were easily rinsed away by agitation with three changes of water and the residue was allowed to dry out in a fume chamber.

\subsection{Determination of Fiber Dimensions and Tensile Strength}

We tested the tensile strength of the fibers using a United LCH-10 speedy tester United Calibration Corporation. For comparison we tested the tensile strength of a typical cotton thread used in the lab and its strength was about $10 \mathrm{lbs}$.

Laser diffraction analyses were also conducted on the fibers by directing a green laser beam $(532 \mathrm{~nm}$ wavelength) on the fiber strands, and the beam allowed to interfere with itself to produce an interference pattern. The distance between adjacent minima was used to determine fiber thickness, using the following formula

$$
d=\frac{\lambda l}{\Delta y}
$$

$\lambda=$ wavelength of laser beam

$l=$ distance from the fiber to the screen

$\Delta y=$ distance between adjacent minima 


\section{Results and Discussion}

The microbial retted fibers differed significantly in color from the chemically retted fiber. While microbial retting yielded slightly green fibers due to the presence of residual plant materials, the chemically retted fibers, as would be expected were much whiter and of softer consistency. However, the tensile strength of the chemically retted product was much lower than the microbial retted fibers. Microbial retting yielded fibers of an average tensile strength of $357 \mathrm{~N}$ (80 Ibs), the chemically retted fibers of comparable diameter showed a tensile strength of 89 $\mathrm{N}$ (20 Ibs). For comparison, a typical cotton thread commonly used in the lab had a tensile strength of about 10Ibs. The microbial retted fiber was, therefore, four times as strong as chemically retted fiber and 8 times as strong as a cotton fibers used as reference. Fiber quality is known to be dependent on the method of extraction, no matter the source of the fiber [4]. While some methods, for example, enzymatic retting could have their advantages, the difficulty level is much higher than other methods [5].

The tensile strength of microbially retted and chemically retted fiber bundle (of same thickness and under same loading condition) were: Naturally retted fiber $-357 \mathrm{~N}(80 \mathrm{lbs})$, chemically retted fiber $-89 \mathrm{~N}$ (20 lbs). This should be expected, due to the inevitable degrading effect of the chemicals applied to the raw materials.

Although microbial retting is by far one of the least expensive methods and also produce much stronger fibers than most other methods, it requires a lot more time to carry out. In addition, it is more difficult to produce clear white color, and this may make the product unattractive for many uses. For example, dyeing will be less successful on the naturally retted microbial product in this work. On the other hand, the superior tensile strength of microbial retted fiber will make it more useful for more strenuous and less sensitive, purposes such as the production of sacs, fish net. The method of choice for producing microbial fibers will ultimately depend on the susceptibility to microbial degradation due to the amount of pectin, cutin and other plant products present on the raw material. For example, the time required to obtain fibers from coconut husks may be too long to be viable. Another important consideration is the final use for which it is intended and the overall cost effectiveness of the process.

Fibers produced by chemical methods

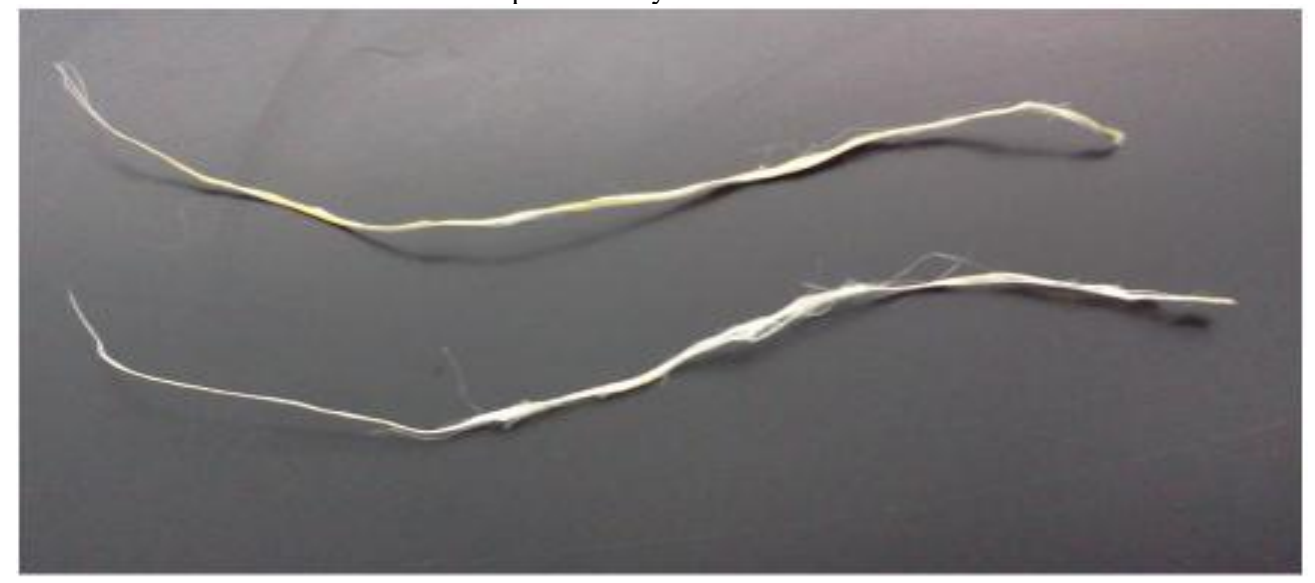

Fig. 1 Samples of chemically retted fibers from Yucca leaves. 
Fibers produces by microbial retting

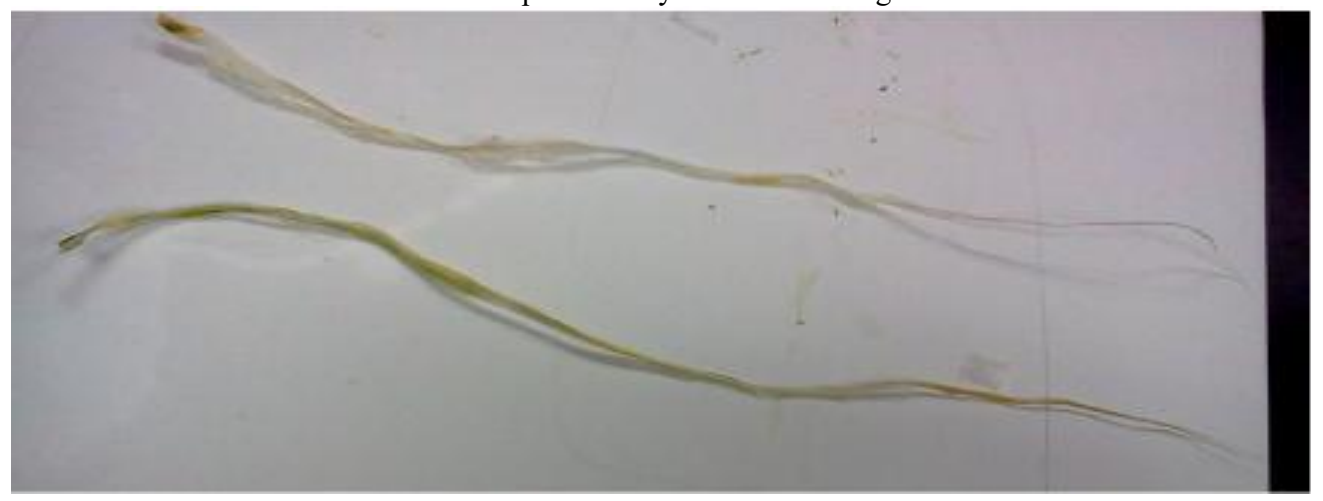

Fig. 2 Samples of microbial retted fibers from Yucca leaves.

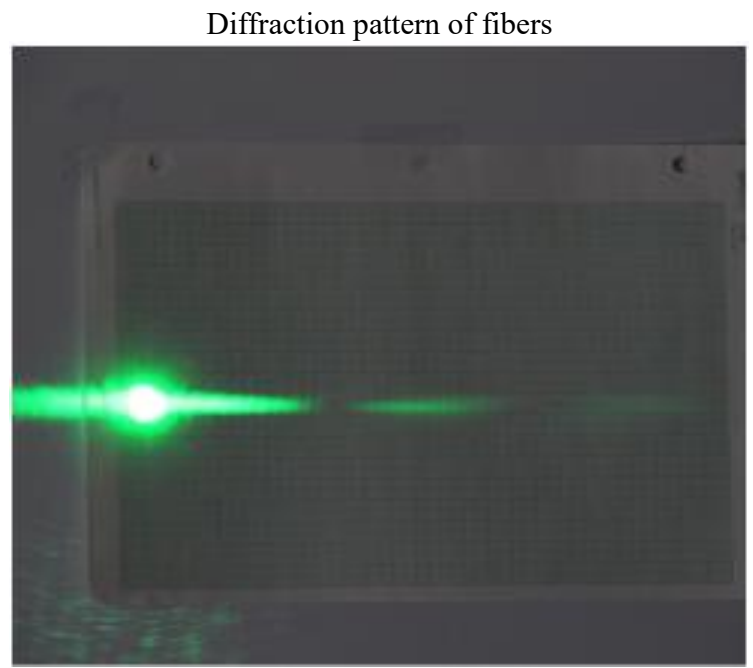

Fig. 3 Fiber diffraction patterns for the determination of fiber dimensions.

It is, therefore, very important to carry out a detailed analysis of available raw materials, economic feasibility as well as the intended use of the final product in order to set up a large scale production of plant fibers for industrial purposes. A combination of methods for retting may be required to obtain fibers which may be intermediate in character and fulfil more variety of purposes. When only microbial retting is desired, it may be useful to carry out much expanded operations, in addition to creating overlapping rounds of retting in order to increase yields in commercial quantities.

\section{References}

[1] Phong, N. T., Fujii T., Chuong, B., and Okubo, K. 2012. "Study on How to Effectively Extract Bamboo Fibers from Raw Bamboo and Wastewater Treatment." Journal of Materials Science Research 1 (1): 144-155.

[2] Naik, K., Swamy, R. P., and Naik, P. 2014. "Design and Fabrication of Areca Fiber Extraction Machine." International Journal of Emerging Technology and Advanced Engineering 4 (7): 860-866.

[3] Reddy N., and Yang, Y. 2009. "Extraction and Characterization of Natural Cellulose Fibers from common Milkweed Stems." Biological Systems Engineering: Papers and Publication, Paper 161, available online at: http://digitalcommons.unl.edu/ biosysfacpub/161.

[4] Paridah, M. T., Basher A. B., ZaifuAzry, S., and Ahmed, Z. 2011. "Retting Process of Some Bast Plant Fibers and Its Effect on Fibre Quality". BioResources 6 (4).

[5] Baracat-Pereira, M. C., Vanetti, M. C. D., de Araujo, E. F., and Silva D. O. 1993. "Partial Characterization of Aspergillus fumigatus Polygalacturonases for Degumming Natural Fibers." Journal of Industrial Microbiology 11: 139-142. 